\title{
Features of Photomechanical Response in Monolayers Composed of a Charged Amphiphilic Azobenzene Polymer
}

\author{
Takahiro Seki,${ }^{\dagger}$ Hidehiko Sekizawa, Ryo-ichi Fukuda, ${ }^{*}{ }^{\dagger \dagger}$ Takashi Tamaki, ${ }^{*}$ \\ Makoto YokoI, ${ }^{* *,+\dagger \dagger}$ and Kunihiro ICHIMURA \\ Research Laboratory of Resources Utilization, Tokyo Institute of Technology, \\ 4259 Nagatsuta, Midori-ku, Yokohama 226, Japan \\ * Department of Molecular Engineering, National Institute of Materials and Chemical Research, \\ 1-1 Higashi, Tsukuba, Ibaraki 305, Japan \\ ** College of Industrial Technology, Nihon University, \\ 1-2-1 Izumi-cho, Narashino, Chiba 275, Japan
}

(Received January 9, 1996)

\begin{abstract}
Monolayers of photochromic azobenzene (Az) containing amphiphilic polymers based on neutral [poly(vinyl alcohol) (PVA)] and cationic [polyallylamine (PAA)] materials were spread on a water surface, and mechanical response induced by photoirradiation was investigated. Observation of photomechanical effects in charged monolayers is reported for the first time. In comparison with the behavior of monolayers of the neutral PVA-based polymer, the monolayer of the cationic PAA derivative was found to show improved photomechanical response in terms of accelerated response and unchanging expanding ability even at higher surface pressures. UV-visible absorption spectroscopic measurements of the monolayers at the air-water interface revealed that the aggregation of the Az side chain of the PAA derivative is more suppressed than the PVA monolayer possibly due to the lateral charge repulsion. Morphological observation by Brewster angle microscopy indicated that the domain structures of the monolayers of PVA- and PAA-based polymers in the trans state are completely different. The neutral monolayer gave rigid and continuous domains without holes, whereas domains of the cationic monolayer exhibited highly amorphous characteristics with numbers of featureless holes. Such differences in the Az packing state can be correlated with variation of the photomechanical response in the two Az monolayers. Addition of a polyanion in the subphase was found to impede substantially the film expansion of the trans PAA-based monolayer due to the interfacial ionic crosslinking.
\end{abstract}

KEY WORDS Azobenzene / Monolayers / Photomechanical Effects / Ionic Polymer / UV-Visible Absorption / Brewster Angle Microscopy /

Polymer gels are now occupying the central position in the research field of stimuli-responsive mechanical materials. ${ }^{1}$ However, in such three dimensional orderless systems, understanding of the macroscopic deformation in terms of molecular processes is extremely difficult. Monolayers restricted in two dimensionality, in this sense, offer intriguing mechanical systems in that macroscopic deformation of materials can be directly coupled with the processes taking place at the molecular level. In two dimensional systems, the arrayed organization aligns the reaction vectors of the individual molecules or units, and markedly simplifies entire processes. It is again anticipated that knowledge obtained in the monolayer systems would offer helpful implications toward understanding the more complicated threedimensional material systems. Besides, a two-dimensional system may find a way to combine completely different mechanical systems of artificial gels which are based on three-dimensional swelling and biological mechanical systems ${ }^{2}$ which involve one-dimensionally directed slipping motions.

Conversion of light energy to mechanical motion can be attained by use of photochromic molecular systems. ${ }^{3}$ To observe reversible photomechanical effect in monomolecular films, two typical photochromic units, spirobenzopyran ${ }^{4-6}$ and azobenzene $(\mathrm{Az})^{7-12}$ have been uti- lized so far. For Az monolayers, Blair et al. ${ }^{7-9}$ studied photomechanical effects in polyamides containing $\mathrm{Az}$ unit in the main chain. For the main-chain type monolayers, the $\mathrm{Az}$ unit is considered to lie flat on the water surface, and exhibit UV-induced contraction probably due to bending of the cis-Az or squeezing deeper into the subphase. More recently, bio-related polypeptides bearing the $\mathrm{Az}$ unit in the side residue were also explored. Malcolm and Pieroni ${ }^{10}$ found UV-light induced contraction of monolayers of an Az-containing poly(Llysine) derivative. Poly(L-glutamate) derivatives having the $\mathrm{Az}$ side chain were investigated by Menzel. ${ }^{11}$ His monolayers, on the contrary, exhibited UV light induced expansion. Since changes in the attractive interaction of the Az moiety with the water surface play an important role (see below), apparently opposing effects reported by the two separate groups may be explained in terms of the difference in the hydrophilicity of the chemical linkage directly connecting the Az unit in the side chain. The poly(L-lysine) derivative of Malcolm and Pieroni ${ }^{10}$ bears an amide group that directly connects the Az unit. This hydrophilic amide group will always be attached to the water surface regardless of the photoisomerized form of the Az unit, and this resembles the situation of the polyamide $\mathrm{Az}$ monolayer. ${ }^{7-9}$

We observed large photomechanical effects in mono-

\footnotetext{
$\dagger$ Corresponding author.

${ }^{\dagger \dagger}$ Present address: Chiyoda Textiles Co., Ltd., Sumida-ku, Tokyo 130, Japan.

${ }^{+\dagger}$ Present address: Toyo Chemical Co., Ltd., Kamakura Kanagawa 247, Japan.
} 

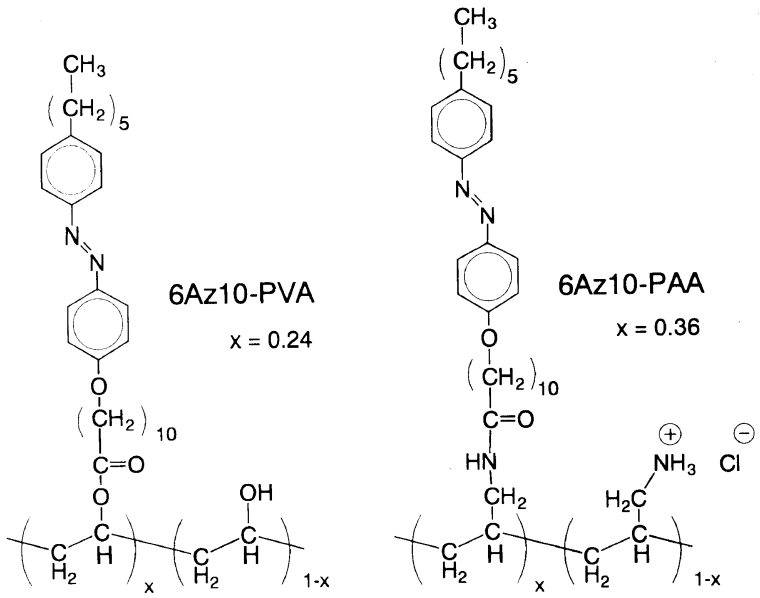

Chart 1.
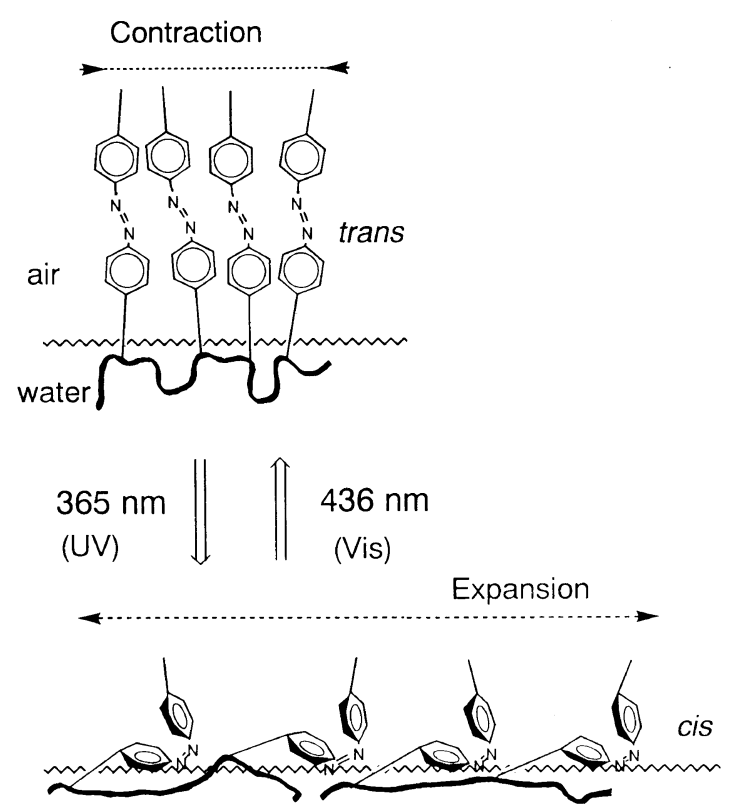

Figure 1. Schematic illustration of UV and visible light induced deformation of the polymeric Az monolayer at the air-water interface. In the process of trans to cis photoisomerization upon UV $(365 \mathrm{~nm})$ light illumination, the $\mathrm{Az}$ unit increases its polarity and experience attraction from the water surface. This movement induces expansion of the entire monolayer. Upon visible $(436 \mathrm{~nm})$ light illumination the reverse process takes place, resulting in photocontraction.

layers composed of poly(vinyl alcohol)s (PVAs) having an $\mathrm{Az}$ side chain. ${ }^{12} \mathrm{~A}$ typical material, 6Az10-PVA, is indicated in Chart 1. These monolayers exhibit area expansion and contraction upon alternative UV (365 nm) and visible $(436 \mathrm{~nm})$ light illumination, respectively. These monolayers behave like Menzel's Az materials. ${ }^{11}$ The film deformation mechanism is considered as follows. The trans to cis photoisomerization caused by UV illumination leads to dipole moment increase from 0.5 to $3.1 \mathrm{D}^{13}$ The polarity increase in the trans to $\mathrm{cis}$ isomerization process gains attraction with the water surface, and consequently the film area expands. The reverse process occurs upon visible light illumination, leading to film contraction. Such behavior is schematically drawn in Figure 1. These photomechanical response processes do not contain complicated relaxation since intercept of the light illumination immediately stops film deformation. ${ }^{12}$ Film area is thus determined by the trans/cis ratio of the existing $\mathrm{Az}$ units in the monolayer. The 6Az10-PVA monolayer shows ca. three-fold area expansion upon UV light illumination, ${ }^{12}$ which is, to our knowledge, the largest photoinduced area changes in monolayers hitherto reported..$^{4-11}$

Our most recent investigation ${ }^{14,15}$ revealed, in comparison with low-molecular-mass materials, that polymeric materials are favorable in mechanical stability giving full reproducibility during repeating cycles. It has also been found that expansion response becomes faster for monolayers in which aggregation of $\mathrm{Az}$ units are prevented more. ${ }^{15}$ The above observations inspired us to introduce a charged group in the main chain to prevent $\mathrm{Az}$ aggregation which is observed in the neutral 6Az10-PVA monolayer. In this context, a cationic polyallylamine (PAA)-based Az amphiphilic material, 6Az10-PAA (Chart 1) was newly prepared, and the photoresponse of this monolayer is examined. Works hitherto investigated concern the photomechanical response in monolayers having neutral backbones. ${ }^{4-12}$ This work deals with, for the first time, the ionic polymer for these observations. In this paper, we wish to report some characteristic photoresponding properties of the charged 6Az10-PAA monolayers in comparison with the behavior of neutral 6Az10-PVA monolayers.

\section{EXPERIMENTAL}

\section{Materials}

Synthetic procedures of 11-[4-[(4'-hexylphenyl)azo]phenoxy]undecanoic acid $(6 \mathrm{Az} 10 \mathrm{COOH})$ and $6 \mathrm{Az} 10$ PVA were described previously. ${ }^{16}$ Poly(styrene sulfonate) sodium salt (PSSNa, $M_{w} \fallingdotseq 70000$ ) was purchased from Aldrich.

The cationic amphiphile 6Az10-PAA was prepared as follows. Three grams of polyallylamine (PAA) hydrochloride salt $(32.1 \mathrm{mmol}$, Toyo Boseki, PAA-HCl-L, $\left.M_{w} \fallingdotseq 1 \times 10^{4}\right)$ were dissolved in methanol $(100 \mathrm{ml})$ and, to this solution, sodium methoxide $(2.6 \mathrm{~g}, 48.2 \mathrm{mmol})$ was added at room temperature. The precipitate was filtered off, and the solvent was evaporated to obtain $1.6 \mathrm{~g}$ of the free base PAA ( $88 \%$ yield).

The condensation reaction of PAA with $6 \mathrm{Az} 10 \mathrm{COOH}$ was performed as follows. Free base PAA $(0.1 \mathrm{~g}, 1.75$ unit mmol) and 1-ethyl-3-(3-dimethylaminopropyl) carbodiimide hydrochloride (water soluble carbodiimide, Kokusan Kagaku, $0.8 \mathrm{~g}, 4.2 \mathrm{mmol}$ ) were dissolved in water $(5 \mathrm{ml})$, and to this solution $6 \mathrm{Az} 10 \mathrm{COOH}(0.65 \mathrm{~g}$, $1.4 \mathrm{mmol})$ dissolved in tetrahydrofuran $(5 \mathrm{ml})$ was added at room temperature. The emulsoidal mixture was stirred at room temperature for $15 \mathrm{~min}$ and then $60^{\circ} \mathrm{C}$ for $1 \mathrm{~h}$. The solution was pored into water $(100 \mathrm{ml})$, and hydrochloric acid aqueous solution was added to $\mathrm{pH} 4$. The resulting precipitate was collected and washed with water. The powder was then dissolved in benzene $(10 \mathrm{ml})$. After an insoluble substance was filtered off, the solution was evaporated and then freeze dried. A yellow powder $(0.45 \mathrm{~g})$ of $6 \mathrm{Az} 10$-PAA was obtained as the hydrochloride salt. The amount of the introduced $\mathrm{Az}$ side chain was estimated to be $36 \%$ of the total unit (see Scheme 1) from photometric measurement using the molar extinction coefficient, $\varepsilon(352 \mathrm{~nm})=3.0 \times 10^{4} \mathrm{dm}^{3} \mathrm{~mol}^{-1} \mathrm{~cm}^{-1}$.

Observation of the spreading behavior and photo- 
mechanical effects in $\mathrm{Az}$ monolayers was performed on a Lauda FW-1 film balance at $21^{\circ} \mathrm{C}$ equipped in a dark room. Photochemically induced area expansion and contraction were observed as follows. The $\mathrm{Az}$ derivative in the trans form dissolved in chloroform $\left(1 \times 10^{-3} \mathrm{~mol}\right.$ $\mathrm{dm}^{-3}$ ) was spread on the subphase at neutral $\mathrm{pH}$. After the solvent was evaporated, the monolayer was compressed to a surface pressure for the area monitor. A UV $(365 \mathrm{~nm})$ or visible $(436 \mathrm{~nm})$ light from a $150 \mathrm{~W}$ high pressure mercury lamp passed through appropriate Corning optical glass filters was irradiated from the top of the water surface. The light beam was spread to allow irradiation of the entire film area in the most expanded state of the monolayers. The exposure energy of UV and visible light was $c a .2 \mathrm{~mW} \mathrm{~cm} \mathrm{~cm}^{-2}$.

UV-visible absorption spectroscopic measurements of floating monolayers were carried out using a multichannel photodiode detector system (Ohtsuka Electronics MCPD-2000) equipped with a $150 \mathrm{~W}$ xenon lamp (Hamamatsu Photonics C4251/C4264, ozone-less type) and optical fibers.

Morphological observation of monolayers at the airwater interface was performed with a home-made Brewster angle microscope (BAM) assembled by Yokoyama and Tabe at Electrotechnical Laboratory. BAM images were monitored and processed by a video recording system equipped with a printer.

\section{RESULTS AND DISCUSSION}

\section{Static Spreading Behavior at the Air-Water Interface}

Figure 2 indicates the surface pressure-area curves of 6Az10-PVA (a) and 6Az10-PAA (b-d) monolayers upon continuous compression at $50 \mathrm{~cm}^{2} \mathrm{~min}^{-1}$ and $21^{\circ} \mathrm{C}$. Monolayers were spread from the trans (solid curve) and UV-irradiated (dotted curve) chloroform solutions. For the UV irradiated solution, content of the $c i$ isomer was approximately $90 \%$ of the total unit in the photostationary state. For 6Az10-PAA, the monolayers were spread on pure water (b), and subphases containing $\mathrm{NaClO}_{4}\left(5 \times 10^{-4} \mathrm{~mol} \mathrm{dm}{ }^{-3}, \mathrm{c}\right)$ and PSSNa $\left(1 \times 10^{-4}\right.$ moldm $\left.{ }^{-3}, \mathrm{~d}\right)$.

The Az monolayers show a common spreading feature that the cis monolayer is highly expanded with some deviations in curve shape. However, clear differences were observed in the trans Az monolayers. The limiting area of trans-Az monolayers estimated by the extrapolation of the steepest slope consistently ranged $0.4-0.5$ $\mathrm{nm}^{2}$, but the trans-6Az10-PAA monolayers without the polyanion were substantially expanded in the low pressure regions compared to that of 6Az10-PVA. The film expansion of 6Az10-PAA can be ascribed to lateral electrostatic repulsion among the ammonium groups in the main chain.

The cis-Az monolayer of 6Az10-PAA on pure water (b) was unstable and exhibited only small surface pressures perhaps due to too large hydrophilicity. Judging from the shape of the cis-Az monolayers of this material, observation of photomechanical effect of 6Az10-PAA monolayer on pure water appeared to be difficult. On the other hand, addition of $\mathrm{NaClO}_{4}$ (c) allowed sufficient surface pressures even in the cis form. This is due to exchanging the counter anion from $\mathrm{Cl}^{-}$to $\mathrm{ClO}_{4}^{-}$. The
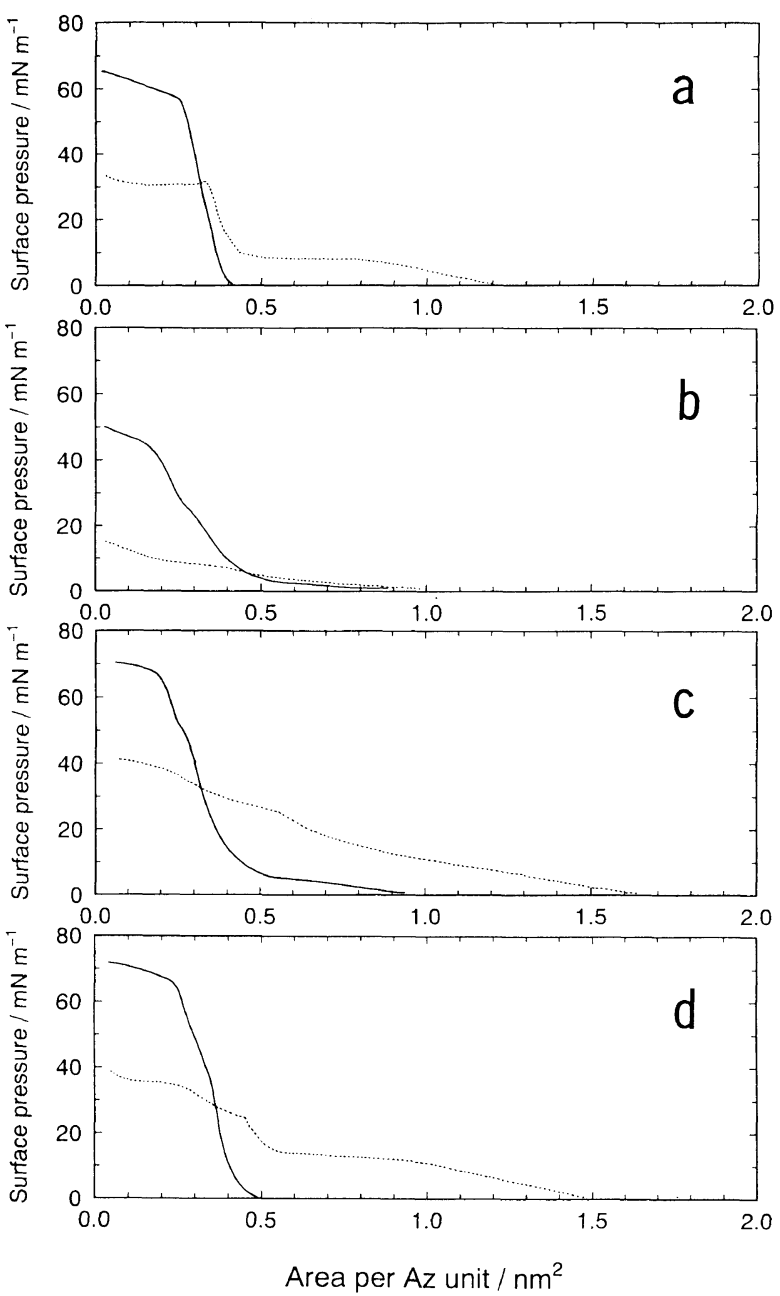

Figure 2. Surface pressure-area curves of 6Az10-PVA (a) and 6Az10-PAA (b-d) monolayers at $21^{\circ} \mathrm{C}$. 6Az10-PAA monolayers were spread on pure water (b), and $\mathrm{NaClO}_{4}\left(5 \times 10^{-4} \mathrm{~mol} \mathrm{dm}{ }^{-3}, \mathrm{c}\right)$ and PSSNa $\left(1 \times 10^{-4}\right.$ unit mol dm $\left.{ }^{-3}, \mathrm{~d}\right)$ containing aqueous solutions. Solid and dotted lines indicate monolayers in the trans and cis ( $c a .90 \%$ contents) form, respectively.

hydrophobic anion forms a closer ion pair and weakens the repulsive electrostatic force, which gains total hydrophobicity. Thus, exploration of photomechanical response on $\mathrm{NaClO}_{4}$ containing subphase seemed more promising. Addition of a polyanion, PSSNa to the subphase further suppressed the expansion of the trans6Az10-PAA monolayer in the low pressure regions (d). The lift off area was less than $0.5 \mathrm{~nm}^{2}$ per Az unit likewise to that of $6 \mathrm{Az} 10$-PVA. The effect of the polyanion will be stated in detail in the last section.

\section{Photoinduced Area Changes of Az Monolayers}

Since the 6Az10-PAA monolayer on pure water was mechanically unstable (see above), observation of photomechanical response was performed on the subphase containing $\mathrm{NaClO}_{4}$.

Alternative UV and visible light illumination induced area changes of 6Az10-PAA monolayers. Figure 3 shows the area response when monitored at $2 \mathrm{mN} \mathrm{m}^{-1}$. The monolayer expanded approximately twice in area upon UV light illumination, and reverted almost to the original area upon visible light illumination. These processes were fully reproducible. Az monolayers com- 


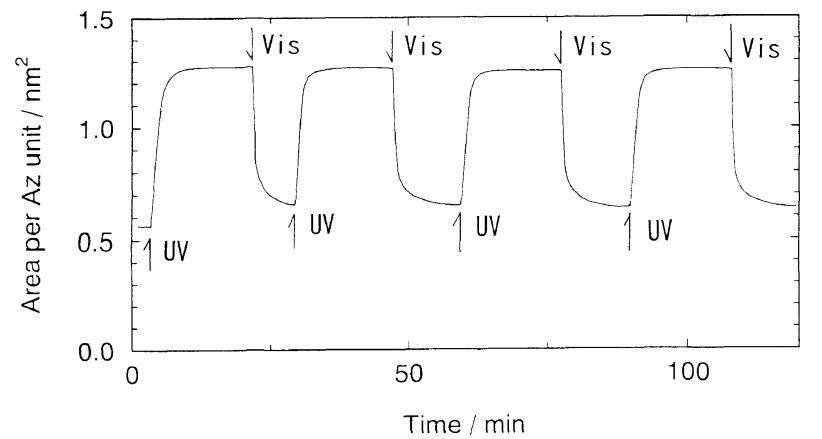

Figure 3. Time profiles of photoinduced deformation of 6Az10-PAA monolayer on the $\mathrm{NaClO}_{4}$ containing $\left(5 \times 10^{-4} \mathrm{moldm}^{-3}\right)$ subphase at $21^{\circ} \mathrm{C}$. The exposure energy of the illumination was $c a .2 \mathrm{~mW} \mathrm{~cm}^{-2}$ at $365 \mathrm{~nm}$ (UV) and $436 \mathrm{~nm}$ (visible) lines.

Table I. UV light induced expansion behavior of Az monolayers ${ }^{\mathrm{a}}$

\begin{tabular}{|c|c|c|c|c|c|c|}
\hline $\begin{array}{c}\text { Monolayer } \\
\text { surface pressure }\end{array}$ & \multicolumn{3}{|c|}{ 6Az10-PVA } & \multicolumn{3}{|c|}{ 6Az10-PAA } \\
\hline $\mathrm{mN} \mathrm{m}^{-1}$ & 2 & 5 & 10 & 2 & 5 & 10 \\
\hline Response time $\mathrm{b}^{\mathrm{b}} / \mathrm{min}$ & 4 & 8 & 10 & 1.5 & 2 & 3.5 \\
\hline Factor of area expansion & 3.1 & 2.8 & 1.3 & 1.9 & 2.0 & 1.8 \\
\hline
\end{tabular}

${ }^{\mathrm{a}} \mathrm{UV}$ irradiation, ca. $2 \mathrm{~mW} \mathrm{~cm}^{-2}$. ${ }^{\mathrm{b}}$ Response time is defined as time required for $90 \%$ change.

posed of low-molecular-mass materials are known to be fatigued after repetitions. The good reproducibility observed here can be, as expected, attributed to the mechanical reinforcing role of polymer chain. The factor of the film expansion for 6Az10-PAA (ca. 2) was less than that for the neutral polymer, 6Az10-PVA (ca. 3). This is a consequence of the expanded state in the trans form due to charge repulsion.

Table I compares the photoresponse properties of 6Az10-PAA and 6Az10-PVA monolayers. Film areas were monitored at 2,5 , and $10 \mathrm{mN} \mathrm{m}^{-1}$. The response time was defined as the time required for $90 \%$ that of total change. With respect to the response time of expansion, 6Az10-PAA monolayers was substantially shortened at all monitoring surface pressures. Another feature can be found in the surface pressure dependence of the factor of area expansion. The expansion factor considerably reduced as the monitoring surface pressure increased for 6Az10-PVA; it decreased from 3.1 to 1.3 when the surface pressure increased from 2 to $10 \mathrm{mN}$ $\mathrm{m}^{-1}$. On the other hand, expansion took place to the same extent for the 6Az10-PAA monolayer. Probably, the above two favorable features stem commonly from charge repulsive force effect in the main chain which prevents side chain arrays from packing closely.

\section{$U V$-Visible Absorption Spectrum and Photoresponse Behavior}

The aggregation state of $\mathrm{Az}$ units can be evaluated by observing the electronic spectrum of the $\pi-\pi^{*}$ absorption band. ${ }^{17}$ The side-by-side aggregation of $\mathrm{Az}$ is characterized by the hypsochromic shift of this band. Therefore, the magnitude of the spectral shift can be an indicator of the lateral packing state of the side chain array. Figure 4 shows the UV-visible absorption spectra of floating monolayers of 6Az10-PVA (a) and 6Az10-

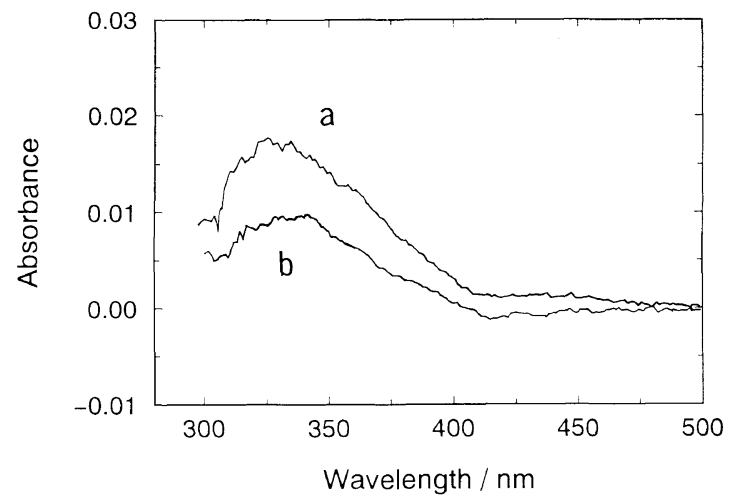

Figure 4. UV-visible absorption spectra of 6Az10-PVA (a) and 6Azl0-PAA (b) monolayers in the trans state on the water surface taken at $2 \mathrm{mN} \mathrm{m}^{-1}$ and $21^{\circ} \mathrm{C}$.

PAA (b) at $2 \mathrm{mN} \mathrm{m}^{-1}$. The $\pi-\pi^{*}$ band of 6Az10-PAA monolayer peaked at $340 \mathrm{~nm}$, which is $10 \mathrm{~nm}$ red-shifted from that of 6Az10-PVA. Since this material in chloroform solution gave the peak maximum at $352 \mathrm{~nm}$, the side-by-side aggregation of Az in 6Az10-PVA monolayer is promoted more. This implies that close packing is suppressed in 6Az10-PAA monolayers due to the charge repulsion. The absorbance of 6Az10-PAA monolayer was approximately half of that for 6Az10-PVA. This is again due to the film expansion of 6Az10-PAA in the lower pressure regions ( $c f$. Figures $2 \mathrm{a}$ and $2 \mathrm{c}$ ).

We previously demonstrated a correlation between the wavelength of the peak top of absorption $\pi-\pi^{*}$ band and UV light induced film expansion behavior for Az containing monolayers. ${ }^{15}$ These materials have a common structure with the exception of the hydrophilic head group structure. In addition to 6Az10-PVA, data for a carboxylic acid derivative $(6 \mathrm{Az} 10 \mathrm{COOH})$ at $\mathrm{pH} 2$, 7 (pure water and $\mathrm{Cd}^{2+}$ containing subphase), and 12 , and an oxazoline derivative (6Az10-Oxa) were shown. As a rule, the more densely packed Az monolayer, which can be evaluated by the magnitude of the hypsochromic shift of the $\pi-\pi^{*}$ band, expands more slowly. For the factor of expansion, there are some optimum wavelength positions to attain the largest expansion. A fully charged monolayer, $6 \mathrm{Az} 10 \mathrm{COO}^{-}$at $\mathrm{pH} \mathrm{12,} \mathrm{responds} \mathrm{very}$ quickly, but the factor of expansion is apparently reduced because it is already highly expanded in the trans form.

Figures 5 and 6 present the response time of the film expansion and expansion factor, respectively, as a function of the peak top wavelength. The open circles are data obtained in the previous work, ${ }^{15}$ and the closed circles indicate those for 6Az10-PAA obtained in this work. It is clear that the plot for 6Az10-PAA monolayer is positioned almost within the above relationship for both properties. The response time of 6Az10-PAA monolayer is comparable to that of $6 \mathrm{Az} 10 \mathrm{COO}^{-}$at $\mathrm{pH}$ 12. The degree of film expansion of 6Az10-PAA monolayer is intermediate of the neutral 6Az10-PVA or 6Az10-Oxa and fully charged $6 \mathrm{Az} 10 \mathrm{COO}^{-}$monolayer.

\section{Brewster Angle Microscopy}

Morphological observation of monolayers may be performed either by fluorescence microscopy or Brewster angle microscopy (BAM). ${ }^{18,19}$ Since Az monolayers are 


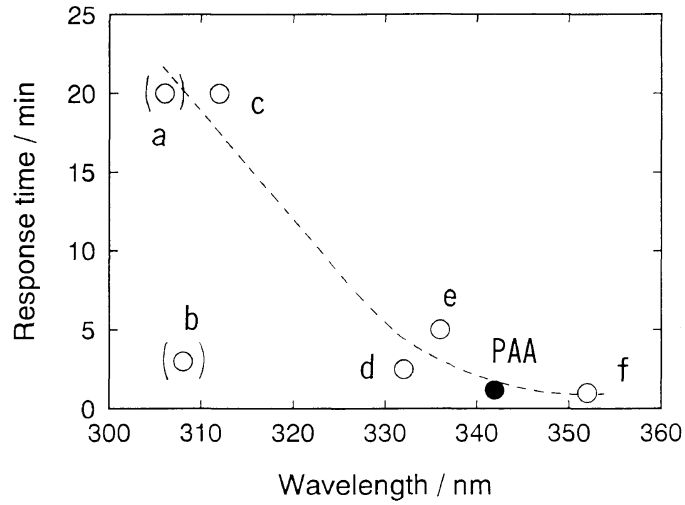

Figure 5. Response time of the UV light induced expansion as a function of the peak position of $\pi-\pi^{*}$ absorption band of $\mathrm{Az}$ unit in the floating monolayers. For definition of response time, see the footnote of Table I. The open circles indicate data obtained in the previous work. ${ }^{15}$ Spread monolayers are $6 \mathrm{Azl} 10 \mathrm{COOH}$ on $\mathrm{Cd}^{2+}$ containing subphase (a), 6Az10COOH at $\mathrm{pH} 2$ (b), 6Az10COOH at pH 7 (c), 6Az10-PVA (d), 6Az10-Oxa (e), and 6Az10COOH at pH 12. For designations of compounds, see the text. The datum of 6Az10PAA in this study is indicated as the closed circle. Data of a and b are indicated in parentheses because the expansion is too small to evaluate the response time properly.

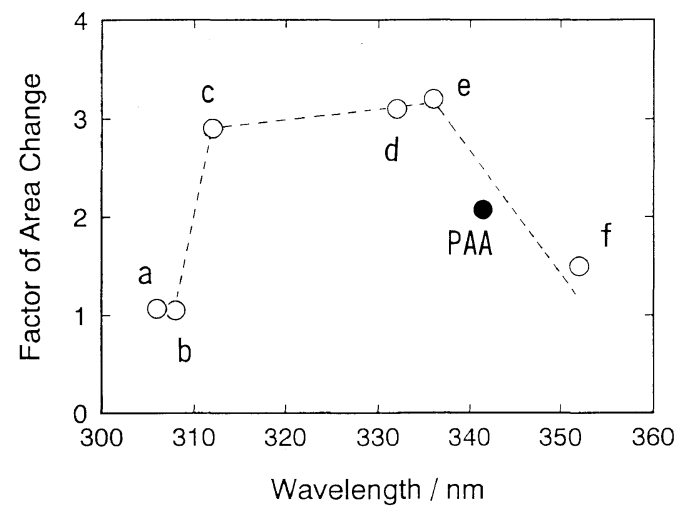

Figure 6. The factor of the area expansion upon UV light illumination as a function of the peak position of $\pi-\pi^{*}$ absorption band of Az unit in the floating monolayers. The notations (a-f) are the same as indicated in the Figure 5.

not fluorescent, the latter optical method is suited for the present monolayer systems. BAM observation of 6Az10-PAA and 6Az10-PVA monolayers in the trans form was carried out as shown in Figure 7. The microscopic observation was carried out at $0 \mathrm{mN} \mathrm{m}^{-1}$ where morphological features of the floating domains could be clearly recognized. The dark and bright regions correspond to the water surface and floating Az containing monolayers, respectively.

Iceberg like domain structures were observed for 6Az10-PVA monolayer showing almost linear shaped boundaries (a). Within the domains the monolayer was continuous without holes. These boundaries seemingly originate from cracking of rigid domains. In contrast, the 6Az10-PAA monolayer exhibited many featureless holes in the monolayer domains (b). The boundaries were not linearly shaped but complicated in outline. Such monolayer morphology is likely to stem from highly amorphous packing state of the $\mathrm{Az}$ side chain. These morphological observations are in agreement with the results of the absorption spectrum measurement of $\mathrm{Az}$ unit (see above). Both BAM and spectroscopic

Polym. J., Vol. 28, No. 7, 1996
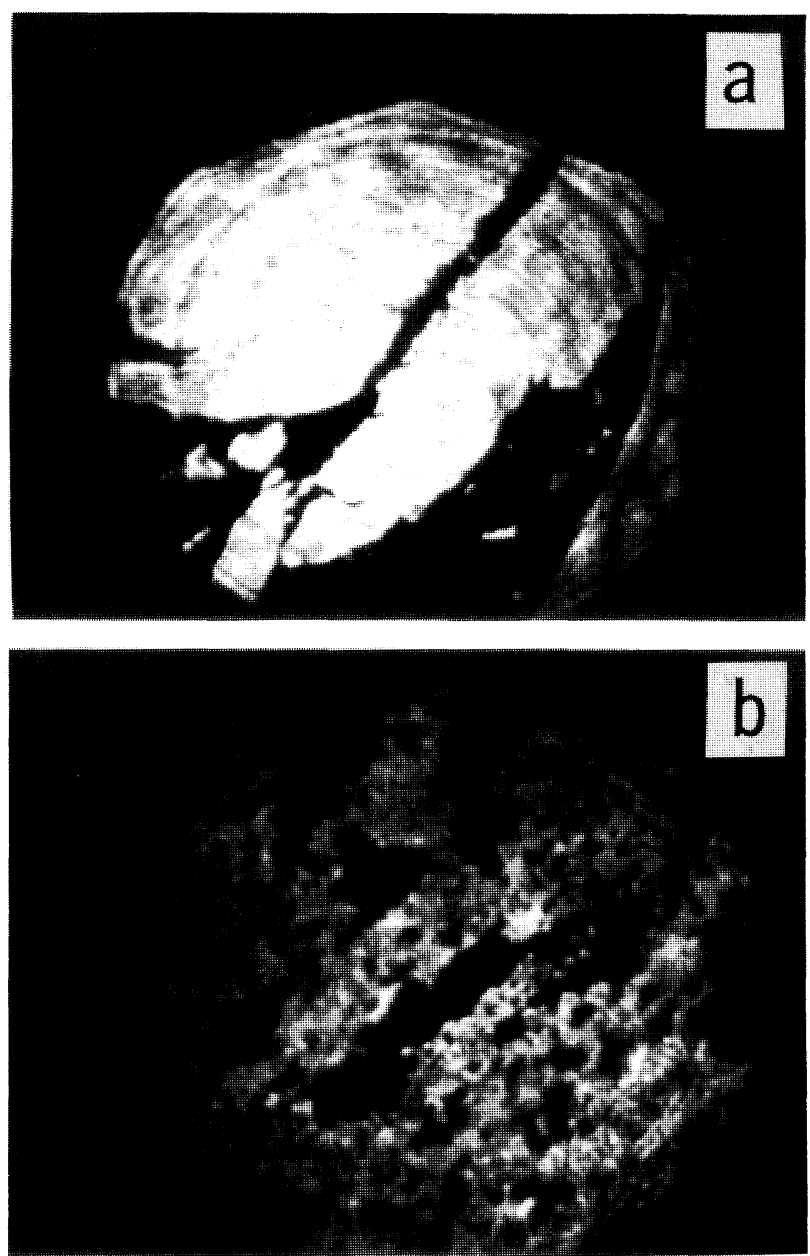

Figure 7. BAM images of 6Az10-PVA (a) and 6Az10-PAA (b) monolayers in the trans form at $0 \mathrm{mN} \mathrm{m}^{-1}$ on water surface. The diameter of the whole circular microscope field is $0.6 \mathrm{~mm}$.

observations prove the loose packing nature of 6Az10PAA. These features can be reasonably coupled with the efficient film expansion upon UV light illumination.

\section{Effects of Ionic Crosslinking with a Polyanion}

Cationic monolayers are known to form interfacial polyion complexes with anionic polyelectrolytes dissolved in the subphase. ${ }^{20-23}$ Such interfacial complexation is anticipated to modify photomechanical response.

In the surface pressure-area curves ( $c f$. Figure 2, c and d) of trans-6Az10-PAA monolayers, addition of PSS $^{-}$ substantially suppressed film expansion below $5 \mathrm{mN} \mathrm{m}^{-1}$, indicating complexation between the cationic 6Az10PAA monolayer and $\mathrm{PSS}^{-}$. The ionic complexation, furthermore, varied some features of the compression curves of cis-6Az10-PAA in the slope in the expanded region above $0.5 \mathrm{~nm}^{2}$ per $\mathrm{Az}$ unit, and the position of the kink around $0.5 \mathrm{~nm}^{2}$ which shifted to smaller area by ca. $0.1 \mathrm{~nm}^{2}$.

Figure 8 compares the behavior of photoinduced deformation of 6Az10-PAA monolayers on subphases containing $\mathrm{ClO}_{4}^{-}$and $\mathrm{PSS}^{-}$. UV light illumination induced expansion for both monolayers, but the magnitude of expansion was substantially smaller for the polyion complexed monolayer. Thus, addition of PSS was found to suppress the expansion of trans 6Az10PAA monolayer. This reduction in dynamic expansion 


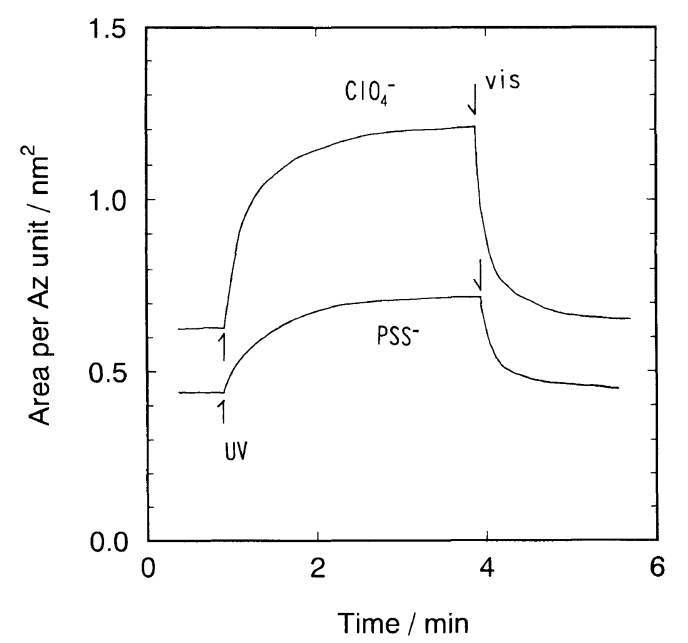

Figure 8. Time profiles of photoinduced deformation of 6Az10-PAA monolayer on $\mathrm{ClO}_{4}^{-}\left(5 \times 10^{-4} \mathrm{~mol} \mathrm{dm}^{-3}\right)$ and $\mathrm{PSS}^{-}\left(1 \times 10^{-4}\right.$ unit moldm ${ }^{-3}$ ) containing subphase at $21^{\circ} \mathrm{C}$. Exposure conditions are the same as in Figure 3.

can be regarded as a consequence of the area fixation of the trans-6Az10-PAA monolayer through the ionic crosslinking by the polyanion. In contrast, a highly expanded area was preserved when a pre-isomerized cis-6Az10-PAA monolayer was spread on $\mathrm{PSS}^{-}$containing subphase from the beginning (see Figure $2 \mathrm{~d}$, dotted line). Therefore, the effect of polyanion was dependent on the initial state of the monolayer. It is thus concluded that the photomechanical response of the cationic Az monolayer can be modified by a negatively charged polyelectrolyte additive.

\section{SUMMARY}

The photomechanical response of monolayers of the cation containing 6Az10-PAA was observed in comparison with those of the homologous neutral 6Az10-PVA. The followings are deduced and concluded.

(i) The UV light induced expansion process is accelerated.

(ii) Magnitude of the film expansion is insensitive to monitoring surface pressure up to $10 \mathrm{mN} \mathrm{m}^{-1}$.

(iii) Above properties can be reasonably correlated with a loose packing of the $\mathrm{Az}$ side chain due to the charge repulsion as proven by $\mathrm{UV}$-visible spectroscopic measurements.

(iv) Microscopic morphology of the trans 6Az10-PAA monolayer is completely different from that of $6 \mathrm{Az} 10$ -
PVA.

(v) Addition of anionic polyelectrolyte suppresses the expansion of trans 6Az10-PAA monolayer due to ionic crosslinking at the interface.

In conclusion, the importance of molecular designing of the main chain structure in the photomechanical response and in the morphology of the Az monolayers is demonstrated.

Acknowledgment. We thank Drs. H. Yokoyama and Y. Tabe at Electrotechnical Laboratory (AIST, Tsukuba) for kindly assembling the Brewster angle microscope.

\section{REFERENCES AND NOTES}

1. D. De Rossi, K. Kajiwara, Y. Osada, and A. Yamauchi, Ed., "Polymer Gels-Fundamentals and Biomedical Application," Plenum Press, New York, N.Y., (1991).

2. M. Irie, Adv. Polym. Sci., 94, 27 (1990).

3. For example: F. Oosawa, Ed., "Advanced Biophysics," 26, 1990.

4. H. Gruder, R. Vilanove, and F. Rondelez, Phys. Rev. Lett., 44, 590 (1980)

5. R. Vilanove, H. Hervet, H. Gruder, and F. Rondelez, Macromolecules, 16, 845 (1983).

6. I. Panaiotov, S. Taneva, A. Bois, and F. Rondelez, Macromolecules, 24, 4250 (1991)

7. H. S. Blair and H. I. Pogue, Polymer, 20, 55 (1979).

8. H. S. Blair, H. I. Pague, and J. E. Riordan, Polymer, 21, 1195 (1980).

9. H. S. Blair and C. B. McArdle, Polymer, 25, 1347 (1984).

10. B. R. Malcolm and O. Pieroni, Biopolymers, 29, 1121 (1990).

11. H. Menzel, Macromol. Chem. Phys., 195, 3747 (1994).

12. T. Seki and T. Tamaki, Chem. Lett., 1739 (1993).

13. D. J. W. Bullock, C. W. N. Cumper, and A. I. Vogel, J. Chem. Soc., 5316 (1965).

14. T. Seki, K. Ichimura, R. Fukuda, and T. Tamaki, Thin Solid Films, in press.

15. T. Seki, R. Fukuda, T. Tamaki, M. Yokoi, and K. Ichimura, Bull. Chem. Soc. Jpn., in press.

16. T. Seki, M. Sakuragi, Y. Kawanishi, Y. Suzuki, T. Tamaki, R. Fukuda, and K. Ichimura, Langmuir, 9, 211 (1993).

17. M. Shimomura, R. Ando, and T. Kunitake, Ber. Bunsenges. Phys. Chem., 87, 1134 (1983).

18. D. Hönig and D. Möbius, J. Phys. Chem., 95, 4590 (1991).

19. S. Henon and J. Meunier, Rev. Sci. Instrum., 62, 936 (1991).

20. M. Shimomura and K. Kunitake, Thin Solid Films, 132, 243 (1985).

21. K. Nishiyama, M. Kurihara, and M. Fujihira, Thin Solid Films, 179, 477 (1989).

22. T. Kajiyama, L. Zhang, M. Uchida, Y. Oishi, and A. Takahara, Langmuir, 9, 760 (1993).

23. C. Erdelen, A. Laschewski, H. Ringsdorf, J. Schneider, and A. Schuster, Thin Solid Films, 180, 153 (1989). 